\title{
A MB-Pulsed-OFDM System Using Discrete Wavelet Packet Transform for Short Range Indoor Wireless Environment
}

\author{
Ch. Sasmita Das, Bikramaditya Das, and Susmita Das
}

\begin{abstract}
Multiband Orthogonal Frequency Division Multiplexing (MB-OFDM) approach using UWB signals with short duration of pulses provide unique opportunities in short-range high data rate wireless applications possessing easy penetration capability through obstacles, high precision ranging and low processing power. Discrete Wavelet Packet Transform (DWPT) reduces inter carrier interference (ICI) and narrowband interference (NBI) compared to FFT based MB-OFDM UWB, offering lower side lobes in the transmitted signal. Performance study of different data rates of DWPT MB-OFDM is attempted for NLOS ultra wideband (UWB) channel in WPAN physical layer standard. In this paper a comparison of DWPT MB-OFDM and DWPT MB-pulsed-OFDM UWB is carried out on a IEEE 802.15.3a channel model through simulation. In LOS channel medium (CM1), at low data rate $(55 \mathrm{Mbps})$, performance of the DWPT MB-pulsed-OFDM system is better due to its inherent frequency diversity. At high data rate of 200 and $480 \mathrm{Mbps}$, the BER performance outperforms than DWPT MB-OFDM. For both low and high data rate applications, DWPT MB-pulsed-OFDM outperforms the conventional FFT MB-OFDM and DWPT MB-OFDM.
\end{abstract}

Index Terms-UWB, MB-OFDM, DWPT MB-OFDM, DWPT MB-pulsed-OFDM, IEEE 802.15.3a, data rate, bit error rate.

\section{INTRODUCTION}

In 2002, the Federal Communications Commission (FCC) allocated a large spectral mask from $3.1 \mathrm{GHz}$ to $10.6 \mathrm{GHz}$ for unlicensed use of commercial UWB communication devices [1], [2]. Since then, UWB systems have gained high interest in both academic and industrial research community. UWB was first used to directly modulate an impulse like waveform with very short duration occupying several GHz of bandwidth [3], [4]. 'Multi-banding' consists in dividing the available UWB spectrum into several sub-bands, each one occupying approximately $500 \mathrm{MHz}$ (minimum bandwidth for a UWB system according to FCC definition) [5]. By interleaving symbols across different sub-bands, UWB system can still maintain the same transmit power as if it was using the entire bandwidth. Narrower sub-band bandwidths also relax the

Manuscript received August 30, 2012; revised February 24, 2014.

Choudhury Sasmita Das is with the Dept. of Electronics and Telecommunication Engineering, P.I.E.T, Rourkela, India (e-mail: ch.sasmitadas@gmail.com).

Bikramaditya Das is with the Department of Electronics and Telecommunication Engineering, VSS University of Technology, Burla, India (e-mail: bikramaditya.vssut@gmail.com).

Susmita Das is with the Electrical Engineering Department, National Institute of Technology Rourkela, India (e-mail: sdas@ nitrkl.ac.in). requirement on sampling rates of $\mathrm{ADCs}$ consequently enhancing digital processing capability.

Multiband-OFDM (MB-OFDM) is one of the promising candidates for PHY layer of short-range high data-rate UWB communications [6]. It combines Orthogonal Frequency Division Multiplexing (OFDM) with the above multi-band approach enabling UWB transmission to inherit all the strength of MB-OFDM technique which has already been proven for wireless communications. The wavelet based MB-OFDM or Wavelet Packet Modulation (WPM) is an alternate approach to conventional MB-OFDM that exploits the self and mutual orthogonality properties of wavelet packet basis functions. Unlike the traditional FFT MB-OFDM which divides the whole bandwidth into several orthogonal and overlapping subbands of equal bandwidths, WPM uses discrete wavelet packet transform to multiplex transmission. DWPM improves spectral efficiency due to the exclusion of CP. Nevertheless, it requires an efficient equalization technique to counter the ISI and ICI [7].

The paper is organized as follows. In Section II we description of the MB-OFDM system model is given. Section III and Section IV discussed Discrete Wavelet Packet Transform (DWPT) MB-OFDM and DWPT MB-pulsed-OFDM respectively. Simulation results are shown and analyzed in Section V. Section VI concludes the paper.

\section{PROCEDURE MB-OFDM SYSTEM MODEL}

The multi-band OFDM system is an OFDM solution proposed for the UWB WPAN physical layer standard. In that proposal, the whole available ultra wideband spectrum between 3.1-10.6 GHz is divided into several sub-bands with smaller bandwidth. The bandwidth of each sub-band is larger than $500 \mathrm{MHz}$ in compliance with the FCC rules for UWB transmission. Specifically, the proposal uses $528 \mathrm{MHz}$ sub-bands. Fig. 1 shows the band planning for the multi-band OFDM system [5]. In each sub-band a normal. OFDM modulated signal with $N=128$ sub-carriers and QPSK modulation is used. In the current proposal, there are four groups of 3-band systems to support 4 independent piconets.

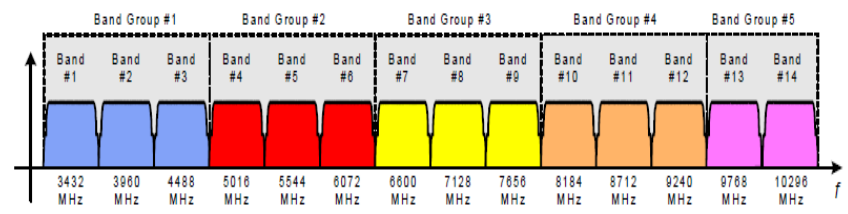

Fig. 1. Band planning for the multi-band OFDM system. 
The main difference between the multi-band OFDM system and other narrowband OFDM systems is the way that different sub-bands are used in the system. The transmission is not done continually on all sub-bands. Rather, it is time multiplexed between different bands in order to use a single hardware for communications over different sub-bands. Different patterns of sub-band switching can be chosen in order to support more piconets operating in the same environments. The OFDM modulation is performed by an inverse fast Fourier transform (IFFT) and a cyclic prefix (CP) is added to cancel inter-block interference (IBI) and inert-channel interference (ICI). A guard interval of silence is also added to allow the transmitter and receiver to switch from one sub-band to another. The signal is then is fed into a D/A converter and sent to the RF section. At the receiver the signal is sampled after down conversion and filtering. Demodulation is performed using a fast Fourier transform (FFT) followed by one-tap frequency domain equalization and decision.

A block of transmit data is scrambled, encoded, interleaved, and quaternary phase shift keying (QPSK)-modulated to form each OFDM symbol [5]. The interleaving is performed using a three-stage block interleaver. In the first stage, data corresponding to three or six subsequent OFDM symbols are interleaved, and the output is partitioned into three or six blocks. In the second stage, each block of the symbol interleaver output is interleaved separately. In the third stage, each symbol is cyclically shifted by a different amount. The interleaved data are modulated by a Gray-mapped QPSK.

TABLE I: SUPPORTABLE DATA RATES FOR MB-OFDM

\begin{tabular}{|c|c|c|c|c|}
\hline Data Rate & Modulation & Code rate & TRF & FRF \\
\hline $55 \mathrm{Mbps}$ & QPSK & $11 / 32$ & 2 & 2 \\
\hline $200 \mathrm{Mbps}$ & QPSK & $5 / 8$ & 1 & 2 \\
\hline $480 \mathrm{Mbps}$ & QPSK & $3 / 4$ & 1 & 1 \\
\hline
\end{tabular}

An inverse FFT (IFFT) is performed on a series of QPSK-modulated symbols to get a series of time domain samples. The total number of subcarriers for OFDM modulation in each subband is 128 , among which 100 are dedicated to data subcarriers, 12 to pilot subcarriers, and 10 to guard subcarriers. The guard subcarriers are in two sets of five, one at each end of the OFDM symbol, with the five subcarriers in each set repeating the nearest five data subcarriers. The remaining 6 subcarriers are nulls over which no signal is transmitted. After the IFFT, a null suffix of 37 samples is attached to the 128 time-domain samples to form a complete OFDM symbol.

The resulting OFDM symbol is 165 samples or $312.5 \mathrm{nsec}$ long, and it is transmitted through a subband determined by the FH pattern. Time and/or frequency domain repetitions are also employed in the OFDM symbol level to enhance the performance. The combinations of the code rate and time/frequency repetition factors (TRF/FRF) determine a set of three supportable data rates, as tabulated in Table I.

\section{DWPT MB-OFDM SYSTEM MODEL}

A signal may be represented by a selected set of wavelet packets without using every wavelet packet for a given level of resolution. The construction of a wavelet packet basis starts from a pair of quadrature mirror filters, $g_{1}$ and $g_{0}$, satisfying the following three conditions [8];

$$
\begin{gathered}
\sum_{n=-\infty}^{\infty} g_{1}(n)=2 \\
\sum_{n=-\infty}^{\infty} g_{1}(n) g_{1}(n-2 k)=2 \delta(k) \\
g_{0}(n)=(-1)^{n} g_{1}(L-n-1)
\end{gathered}
$$

The sequence of functions $\phi_{n}(x)$, called wavelet packets, are recursively defined by quadrature mirror filters $g_{1}(n)$ and $g_{0}(n)$, as

$$
\begin{aligned}
\phi_{2 n}(x) & =\sum_{k \in z} g_{1}(k) \phi_{n}(2 x-k) \\
\phi_{2 n+1}(x) & =\sum_{k \in z} g_{0}(k) \phi_{n}(2 x-k)
\end{aligned}
$$

The two operators also known as filtering down sampling processes using the QMF $g_{1}(n)$ and $g_{0}(n)$ are defined as:

$$
\begin{aligned}
& G_{1}\{x\}(2 n)=\sum_{k \in z} x(k) g_{1}(k-2 n) \\
& G_{0}\{x\}(2 n)=\sum_{k \in z} x(k) g_{0}(k-2 n)
\end{aligned}
$$

These two operators are used to decompose any discrete function $x(n)$ on the space $l^{2}(Z)$ into two orthogonal subspaces $l^{2}(2 Z)$. In each step two coefficient vectors has a length half of the input vector are produced. Thus, the total data length remains unchanged. The process continues and stops at any desired step. The output coefficient vectors become scalars for the deepest decomposition level. This decomposition process is named as Discrete Wavelet Packet Transform (DWPT) [8], [9]. The transformed coefficient vectors are orthogonal and the original signal $x(n)$ can be recovered from the coefficient vectors by the inverse transform. The wavelet packets function set defined in Eq. (4) and Eq. (5) can also be constructed using the Inverse DWPT (IDWPT) with the dual operators of Eq. (6) and (7) are defined as:

$$
\begin{aligned}
& G_{1}^{-1}\{x\}(2 n)=\sum_{k \in z} x(k) g_{1}(n-2 k) \\
& G_{0}^{-1}\{x\}(2 n)=\sum_{k \in z} x(k) g_{0}(n-2 k)
\end{aligned}
$$

The process of constructing a wavelet packet function set can be seen via three levels of wavelet packet tree as shown in Fig. 2 [9].

The good frequency characteristics and greater flexibility of wavelet packet transform make it a choice for MB-OFDM. Fig. 3 shows the transmitter and receiver part of DWPT MB-OFDM. This differs from the conventional MB-OFDM in the sense that IFFT and FFT block is replaced by IDWPT and DWPT respectively. It can also be seen that cyclic prefix block has been excluded due to use of discrete wavelet packet transform, so this increases the spectral efficiency compared to conventional MB-OFDM. In DWPT MB-OFDM 
transmitter the data symbols are converted from serial to parallel and then transmultiplexed by IDWPT block. At the receiver part the data is converted from serial to parallel and then discrete wavelet packet transform is performed. The suggested discrete wavelet based MB-OFDM improve BER performance of transreceiver. The simulation results of implementation of DWPT MB-OFDM transreceiver in UWB CM3 CM4 channel model is shown in next sections.

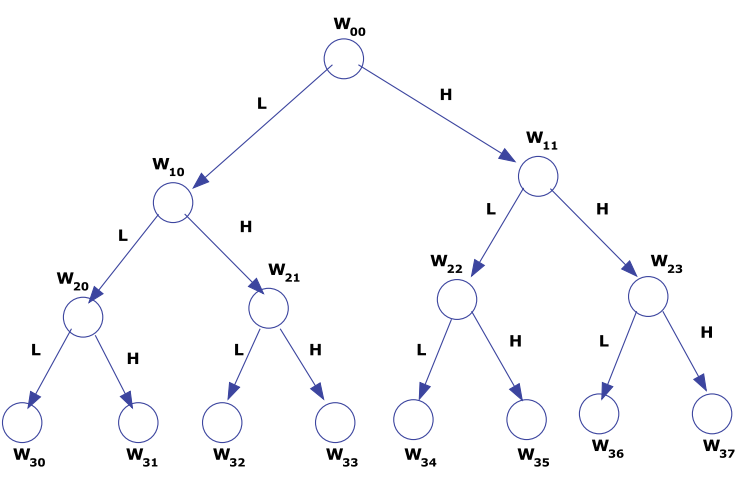

Fig. 2. Three levels of wavelet packet tree.

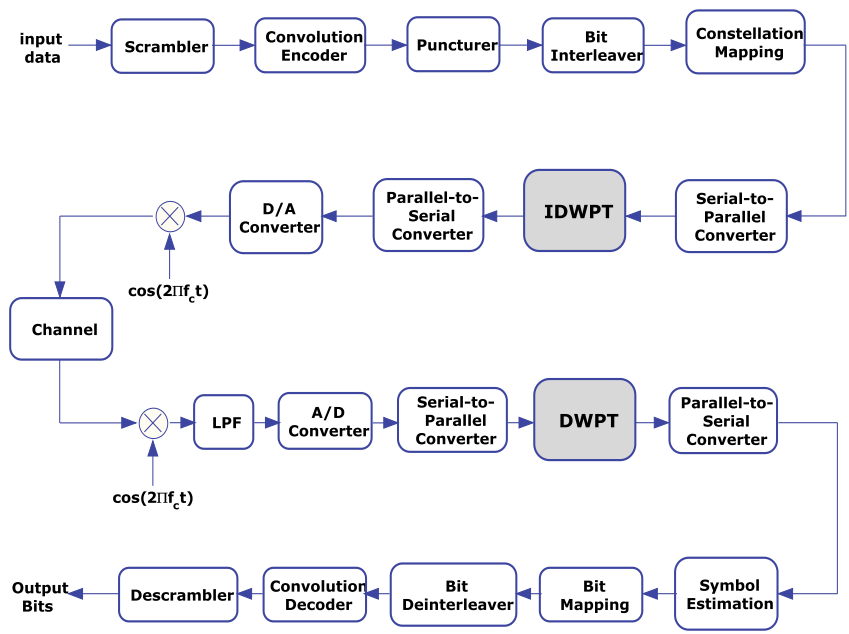

Fig. 3. Block diagram of DWPT MB-OFDM system.

\section{DWPT MULTIBAND (MB)-PULSED-OFDM}

Pulsed MB-OFDM uses orthogonal pulsed subcarriers and generated by up-sampling the digital OFDM symbol after IDWPT block. The up-sampled signal is then passed through a D/A converter and sent over the channel medium. Pulsed OFDM enhances the system performance by providing $k$ diversity branches which can be combined together using any diversity technique in dense multipath UWB channel. Where $k$ is the redundancy factor in pulsed DWPT MB-OFDM system

\section{SimUlation STUdy AND ANALYSIS}

As mentioned before, the case of UWB channels CM3 and CM4 [10] were studied. The performance of the MB-OFDM based PHY layer is evaluated over different modified realistic indoor UWB channel scenarios as defined in the previous sub-section. This mode employs three sub bands of $528 \mathrm{MHz}$ (3.1-4.684 GHz). A 128 point IFFT/FFT is used along with a short cyclic prefix (CP) length of $60.6 \mathrm{~ns}$. Also, an additional guard interval of $9.5 \mathrm{~ns}$ is added to allow the transmitter and receiver to switch from one sub-band to another. This results in a total OFDM symbol duration of $312.5 \mathrm{~ns}$ occupying 528 $\mathrm{MHz}$, which is sent through the UWB channel. Table II provides simulation parameters. All simulation results were obtained using a transmission of at least 500 packets with a payload of 1024 bytes each. In our simulations, when there is no diversity (480 Mbps), a one-tap frequency-domain equalizer is used at the receiver, like that of a conventional OFDM system.

TABLE II: SIMULATION PARAMETERS

\begin{tabular}{|l|c|}
\hline Bandwidth & $528 \mathrm{MHz}$ \\
\hline No. of subcarriers & 128 \\
\hline Information length & 242.5 \\
\hline OFDM Symbol length & $312.5 \mathrm{~ns}$ \\
\hline Subcarrier frequency spacing & $4.125 \mathrm{MHz}$ \\
\hline Data transmission rate & $55,480 \mathrm{Mbps}$ \\
\hline Pilot carrier & 500 \\
\hline FFT size & 128 \\
\hline DWPT size & 128 \\
\hline No. of data tones & 100 \\
\hline No. of pilot tones & 12 \\
\hline No. of guard tones & 10 \\
\hline Constellation & QPSK \\
\hline Wavelet & Haar Transform \\
\hline
\end{tabular}

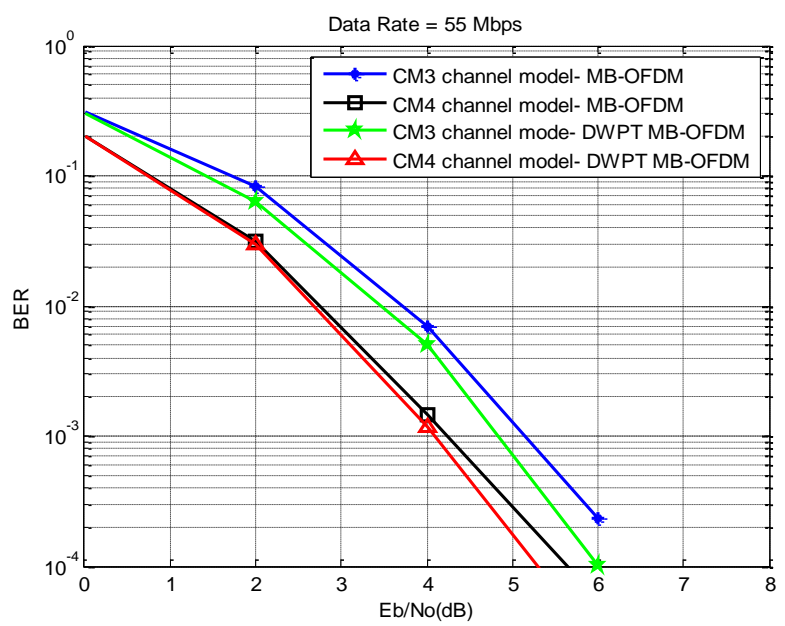

Fig. 4. Performance of MB-OFDM and DWPT MB-OFDM in UWB CM3, CM4 for 55 Mbps data rate.

However, when frequency-diversity is exploited in the system, Maximal Ratio Combining (MRC) technique is used to combine different diversity branches. Then, a soft Viterbi decoder followed by a de-interleaver is used to recover the binary data. As shown in Fig. 4 and Fig. 5, it can be observed that at low data rate (55 Mbps) both MB-OFDM and DWPT MB-OFDM performs better than high data rate of $480 \mathrm{Mbps}$. Further interesting results can be observed at data rate of 55 Mbps. MB-OFDM system performs better in CM4 channel model than CM3 NLOS channel model. Similar performances are observed in case of DWPT MB-OFDM system. Again at low SNR's, the performance of both DWPT MB-OFDM system and MB-OFDM system are almost same. But at high SNR's, DWPT MB-OFDM provides 1dB SNR improvement than MB-OFDM at $10^{-3}$ BER level. A comparison between DWPT MB-OFDM and DWPT-pulsed-MBOFDM in LOS IEEE 802.15.3a channel model is provided in Fig. 6. At $10^{-3}$ 
BER floor, performance of DWPT MB-pulsed-OFDM is almost same at low data rate of $55 \mathrm{Mbps}$. All most 1dB SNR improvement is achieved for the DWPT MB-pulsed-OFDM at $10^{-2}$ BER floor.

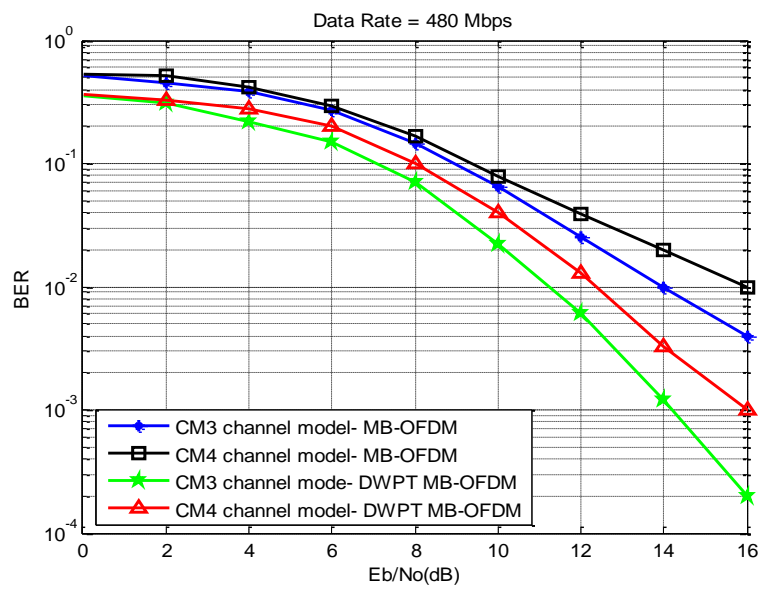

Fig. 5. Performance of MB-OFDM and DWPT MB-OFDM in UWB CM3, CM4 for $480 \mathrm{Mbps}$ data rate.

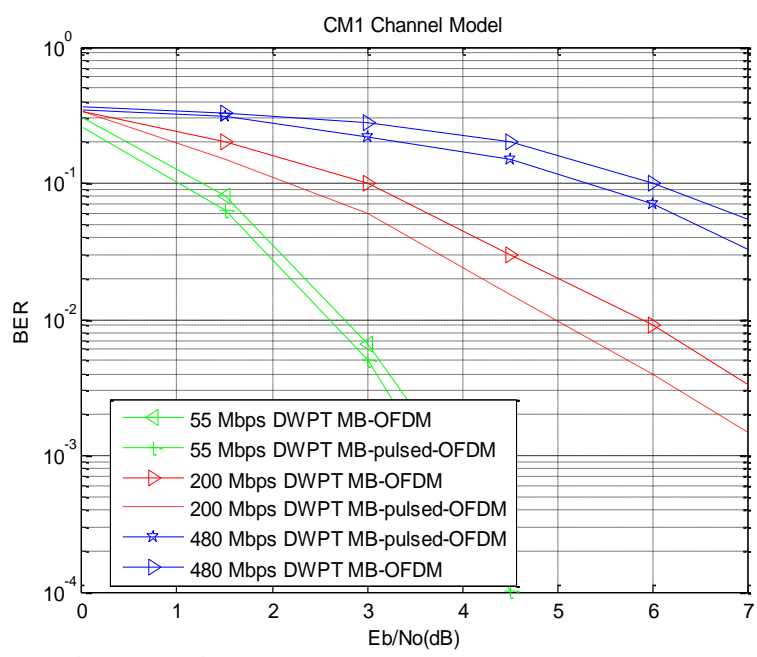

Fig. 6. Performance of DWPT MB-OFDM and DWPT MB-pulsed-OFDM in CM1 channel model, for 55, 200 and $480 \mathrm{Mbps}$ data rate.

As expected with increase in data rate, DWPT MB-pulsed-OFDM and DWPT MB-OFDM has almost same performance in low SNR but performance of pulsed based one is found to be better from medium to high SNR value.

\section{CONCLUSION}

Performance comparison of DWPT MB-pulsed-OFDM system in indoor wireless propagation environment with MB-OFDM at different data rates has been carried out in this research. It is observed from the BER performance curves that the DWPT MB-OFDM system performs better in the CM4 channel environment at $55 \mathrm{Mbps}$. For high data rate i.e, $480 \mathrm{Mbps}$ mode, the performance in CM3 channel model was found to be better than in CM4. Also DWPT improves the spectral efficiency due to the exclusion of cyclic prefix at the start of each symbol unlike the conventional one. As DWPT MB-pulsed-OFDM provides multipath diversity branches as in DWPT MB-OFDM, so the performance remains same for low SNR and improves at high SNR. Thus it can be concluded that both the conventional and pulsed based DWPT
MB-OFDM system provides very good technical solution for UWB PHY layer in short-range high data-rate wireless applications.

\section{REFERENCES}

[1] S. Roy, J. R. Foerster, V. S. Somayazulu, and D. G. Leeper, "Ultrawideband radio design: the promise of high- speed, short-range wireless connectivity," Proceedings of IEEE, vol. 92, issue 2, pp. 295-311, 2004.

[2] M. Z. Win and R. A. Scholtz, "Ultra-wide bandwidth time-hopping spread-spectrum impulse radio for wireless multiple-access communications," IEEE Trans. Comm., vol. 48, issue 4, pp. 679-689, 2000.

[3] B. R. Vojcic and R. L. Pickholtz, "Direct sequence code division multiple access for ultrawide bandwidth impulse radio," in Proc. Military Communications Conference, 2003, vol. 2, pp. 898-902.

[4] S. M. Sajad, A. Mahmood, E. Jaffrot, and P. Duhamel, "Multiband OFDM: a new physical layer proposal of UWB communications," presented at IEEE Conferences, May 2006.

[5] A. Batra, J. Balakrishnan, G. R. Aiello, J. R. Foerster, and A. Dabak, "Design of a multiband OFDM system for realistic UWB channel environments," IEEE Transactions on Microwave Theory and Techniques, vol. 52, issue 9, pp. 2123-2138, 2004.

[6] L. Hanzo, M. Munster, B. J. Chol, and T. Keller, OFDM and MC-CDMA for Broadband Multi-user Communications, WLANs and Broadcasting, John Wiley \& Sons, 2003.

[7] H. J. Taha and M. F. M. Salleh, "Performance comparison of wavelet packet transform (WPT) and FFT-OFDM system based on QAM modulation parameters in fading channels," WSEAS Transactions on Communications, vol. 9, issue 8, pp. 453-462, 2010.

[8] S. Baig, F. Rehman, and M. J. Mughal, "Performance comparison of DFT, discrete wavelet packet and wavelet transforms, in an OFDM transceiver for multipath fading channel," in Proc. 9th International Multitopic Conference, 2005, pp. 1-6.

[9] D. Gupta, V. B Vats, and K. K. Garg, "Performance analysis of DFT-OFDM, DCTOFDM, and DWT-OFDM systems in AWGN channel," in Proc. IEEE Fourth International Conference Wireless and Mobile Communications, Athens, 2008, pp. 214-216.

[10] B. Das and S. Das "Performance study of discrete wavelet packet based MB-OFDM system for short range indoor wireless environment," presented at ICDECOM'11, Ranchi, India.

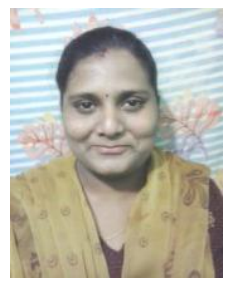

Choudhury Sasmita Das received her M.Tech in electrical engineering with specialization electronics system \& communication from the NIT, Rourkela, Orissa, India, in 2012. Currently she is working as a lecturer in the electronics and telecommunication engineering at the P.I.E.T, Rourkela, India. Her research interests include wireless communication and DSP.

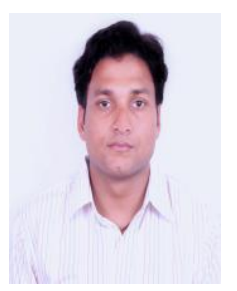

Bikramaditya Das received his B.Tech in electronics and telecommunications engineering from the University of B.P.U.T, Rourkela, Orissa, India, in 2007. From 2008 to 2011 he was a research fellow under the Department of Electrical Engineering at the N.I.T, Rourkela, India. Currently he is working as a lecturer in the Department of Electronics and Telecommunication Engineering at the VSSUT (Formerly UCE), Burla, India. He is an associate member of IEI. His research interests include wireless communication, DSP, control system and robotics etc.

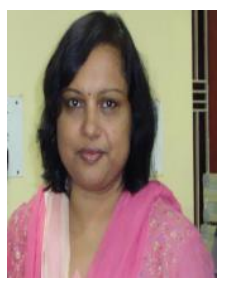

Susmita Das is an associate professor of electrical engineering, NIT, and Rourkela, India. She has twenty years of teaching and research experience and has many research papers to her credit. She is a member of IEEE, fellow IETE, LM ISTE and member of IEI. Her research interests include wireless communication, DSP, and application of soft computing techniques etc. 